\title{
Contemporary management of borderline resectable pancreatic ductal adenocarcinoma
}

\author{
Kyriaki Lekka', Evanthia Tzitzi ${ }^{1}$, Alexander Giakoustidis ${ }^{2}$, Vassilios Papadopoulos ${ }^{1}$, and Dimitrios Giakoustidis ${ }^{1}$ \\ ${ }^{1}$ First Department of Surgery, Medical School, Aristotle University of Thessaloniki, General Hospital Papageorgiou, \\ Thessaloniki, Greece, ${ }^{2}$ Department of HPB Surgery, The Royal London Hospital, London, UK
}

\begin{abstract}
Pancreatic ductal adenocarcinoma (PDAC) remains one of the most aggressive tumors, with a low rate of survival, likely due to the tendency of the tumor for early local and distant spread. Pancreatic cancer accounts for about $3 \%$ of all cancers in the US and about $7 \%$ of all cancer deaths. Surgical resection still represents the best curative treatment for PDAC, although only $10-20 \%$ of patients are upfront resectable at diagnosis, $50 \%$ has metastatic disease and $35 \%$ locally advanced cancer. The 5 -year overall survival (OS) after curative resection is limited to $20 \%$. Moreover among patients who undergo surgery, $30 \%$ develop early recurrence while most of them will eventually relapse. The risk of early failure after surgery could be associated with inadequate preoperative radiological staging, lack of radical surgery and differences in tumor aggressiveness. In recent years, more accurate patient categorization due to sophisticated imaging tools and techniques increase the survival rate while neoadjuvant treatment can help surgeons select patients who will benefit most from surgery. Neoadjuvant therapy includes chemotherapy alone, chemoradiotherapy, chemotherapy with chemoradiation and targeted therapies. The aim of this review is to present the available data concerning the management of patients with borderline PDAC. (Ann Hepatobiliary Pancreat Surg 2019;23:97-108)
\end{abstract}

Key Words: Pancreatic adenocarcinoma; Borderline resectable; Chemotherapy; Chemoradiation

\section{INTRODUCTION}

Pancreatic cancer is currently the 4th leading cause of death by cancer in both women and men in Europe and United States. Pancreatic cancer patients lose $98 \%$ of their healthy life expectancy at the point of diagnosis. ${ }^{1}$ The American Cancer Society's estimates for 2018 indicate that 55,440 people $(29,200$ men and 26,240 women $)$ will be diagnosed with pancreatic cancer while about 44,330 people (23,020 men and 21,310 women) will die of it.

Despite the fact that surgery for PDAC remains the path to prolonged survival, increased attention was brought to the role of adjuvant and neo-adjuvant chemotherapy/ chemoradiation especially in borderline resectable pancreatic cancer (BRPC) in order to increase the respectability rate. Determining resectability of the primary tumor requires appropriate preoperative staging, sophisticated im- aging and multidisciplinary experts in PDAC in order to avoid unnecessary operations and to discriminate unresectable disease from BRPC. ${ }^{2}$

Historically, high-quality computed tomography (CT) scanning has been used to classify PDAC to resectable (stages 1 and 2), locally advanced (stage 3) and metastatic-unresectable tumor (stage 4). In recent years, sophisticated imaging tools and techniques tend to create a new growing category of patients under the term of borderline resectable PDAC. This subgroup includes patients whose disease includes vessel involvement. Although these cases were considered as unresectable tumor in the near past, today indication for curative resection is taken into consideration as the appropriate treatment. ${ }^{2}$

International Study Group for Pancreatic Surgery and National Comprehensive Cancer Network (NCCN) have published special criteria for resectability taking under

Received: August 6, 2018; Revised: January 3, 2019; Accepted: January 20, 2019

Corresponding author: Dimitrios Giakoustidis

First Department of Surgery, Medical School, Aristotle University of Thessaloniki, General Hospital Papageorgiou, Ring Road, Pavlou Mela 56403 Thessaloniki, Greece

Tel: +30-2310992875, Fax: +30-2310237914, E-mail: dgiak@auth.gr

Copyright (C) 2019 by The Korean Association of Hepato-Biliary-Pancreatic Surgery

This is an Open Access article distributed under the terms of the Creative Commons Attribution Non-Commercial License (http://creativecommons.org/ licenses/by-nc/4.0) which permits unrestricted non-commercial use, distribution, and reproduction in any medium, provided the original work is properly cited. Annals of Hepato-Biliary-Pancreatic Surgery - pISSN: 2508-5778 - elSSN: 2508-5859 
consideration the radiographic findings, including CT with a pancreas-specific protocol, the presence or absence of metastasis and the association of tumor with major blood cells. According to NCCN criteria localized PDAC is distinguished as resectable, borderline resectable and unresectable on the basis of distant metastasis and vessels' involvement. $^{3}$

Resectable is the tumor without distant metastasis, without extensions to arterial vessels specifically celiac axis (CA), superior mesenteric artery (SMA) and hepatic artery (CHA) and without contact with superior mesenteric vein (SMV) or portal vein $(\mathrm{PV})$ or $<180^{\circ}$ contact with vein contour irregularity. ${ }^{3}$

Borderline resectable PDAC in the pancreatic head is the solid tumor contact with CHA without extension to CA or artery bifurcation and variant arterial anatomy, contact with $\mathrm{SMA}<180^{\circ}$, contact with $\mathrm{SMV}$ or $\mathrm{PV}<180^{\circ}$ without venous contour irregularity or thrombosis which allows safe and complete arterial and venous resection and reconstruction. ${ }^{3}$

Borderline resectable PDAC in the pancreatic body/tail is the solid tumor contact with the CA of $<180^{\circ}$ or contact with $\mathrm{CA}>180^{\circ}$ without involvement of the aorta and gastroduodenal artery in order to allow a modified Appleby surgery. The above vessel involvement is under discussion because some members of NCCN believe that it is contraindication for resection. ${ }^{3}$

Unresectable ductal adenocarcinoma regarded the tumor with distant metastasis, including non-regional lymph node metastasis. Depended of the anatomy, PDAC is considered unresectable if it is in contact with SMA or CA more than $180^{\circ}$ or has any aortic and venous involvement which cannot be reconstructed surgically. ${ }^{3}$

There is always a risk of early failure after surgery which commonly associated with inadequate preoperative radiological staging, lack of radical surgery and variation in tumor aggressiveness. Recent studies indicate that PDAC appears a heterogeneous spectrum of different biological characteristics which determine tumor progression and its response to treatment. ${ }^{4}$

\section{PANCREATIC CANCER BIOLOGY}

Despite recent progress in treatment of pancreatic cancer, the overall median survival still remains extremely poor, As a result, new curative approaches are urgently needed to be applied. ${ }^{5}$ Remarkably, due to self-sufficiency in growth signals, limitless replication, apoptosis resistance and metastatic invasion, pancreatic tumor cells have decreased response to conventional radio- and chemotherapy. ${ }^{5,6}$ Therefore, substantial research into tumor microenvironment is required to identify targets for developing potential treatment agents. ${ }^{6}$

TGF- $\beta$ seems to be a potential biomarker of tumor activity, as it regulates the epithelial-mesenchymal transition process. ${ }^{6}$ Melisi et al. ${ }^{7}$ demonstrated a phase II study comparing the efficacy of galunisertib, a TGF- $\beta$ inhibitor, and gemcitabine in contrast to gemcitabine alone in patients with unresectable pancreatic cancer. Combination of agents resulted in a higher median overall survival of 10.9 months, compared with 7.2 months for gemcitabine alone, thus leading to tumor suppression, when decreased levels of TGF- $\beta$ were noted. ${ }^{7}$ Overexpression of vascular endothelial growth factor (VEGF) in pancreatic cancer promotes neo-angiogenesis and disorganization of tumor vasculature. ${ }^{6}$ In a double-blind, placebo-controlled, phase III trial, axitinib, a selective inhibitor of VEGF receptors, and gemcitabine, have been administered in patients with locally advanced or metastatic pancreatic cancer. ${ }^{8}$

However, the addition of axitinib to gemcitabine compared to gemcitabine and placebo showed no significant improvement in overall survival. ${ }^{8}$

Recent studies have also focused on therapeutic agents targeting stroma cells. Pancreatic stellate cells (PSCs), also known as activated fibroblasts, play a central role in pancreatic tumor resistance, invasion and progression increasing extracellular matrix synthesis. ${ }^{9}$ All-trans retinoic acid (ATRA), combined with gemcitabine, can diminish PSCs' invasive ability through the RAR- $\beta /$ MLC- 2 pathway, thereby resulting in a more quiescent phenotype. ${ }^{10}$ In addition, paresotide, a somatostatin analogue, combined with gemcitabine, was effectively utilized to inhibit the protein synthesis mTOR/4E-BP1 pathway in cancer-associated fibroblasts, reducing tumor growth and chemoresistance. ${ }^{11}$ Tumor-associated macrophages (TAMs) also play a critical role in tumor microenvironment by suppressing the immune response, promoting tumorigenesis, metastasis and angiogenesis. ${ }^{12}$ Immunotherapies targeting TAMs include therapeutic agents depleting TAMs or inhibiting TAM differentiation, regulating TAM polarization 
or blocking factors related with pro-tumor function of TAMs. $^{12}$

Furthermore, Mahalingam et al. ${ }^{13}$ published a phase II trial investigating the efficacy of reolysin, an oncolytic virus, in combination with gemcitabine, in advanced pancreatic cancer patients. Thirty-four patients were included in the study. Partial response was achieved in 1 of 34 , stable disease was found in 23 of 34, whereas progressive disease was found in 5 of 34 . Median overall survival was estimated to be 10.2 months. Upregulation of programmed death ligand (PD-L1) following reolysin was noted, highlighting the potential of combining oncolytic virus treatment with checkpoint inhibitors. ${ }^{13}$ TNF-related apoptosis-inducing ligand (TRAIL) was referred to induce apoptosis in tumor cells through binding of the trans-membrane death receptors (DR) TRAIL-R1/R2 and DR5. ${ }^{5}$ Recent data of phase I and II studies are available on TRAIL-R agonists antibodies, conatumumab and tigatuzumab, as a promising treatment for pancreatic cancer. ${ }^{5}$ Although the combination of the previously mentioned antibodies with gemcitabine resulted in high percents of stable disease and improved 6-month-survival in patients with metastatic pancreatic cancer, it failed to ensure longterm survival rates and tumor response in comparison with gemcitabine alone. ${ }^{5}$ Further intensified research should be directed towards molecular mechanisms that can suppress or trigger pancreatic tumor cells' proliferation and metastatic invasion and seek for targeted therapy agents.

\section{NEUTROPHIL TO LYMPHOCYTE RATIO (NLR) AND PLATELET TO LYMPHOCYTE RATIO (PLR)}

Prognostic markers for risk stratification in patients with borderline pancreatic cancer would be of significant value to clinicians, especially for those with economical and easily calculated features. Such markers include C-reactive protein (CRP), the Modified Glasgow Prognostic Score, and ratios such as the neutrophil to lymphocyte ratio (NLR) and platelet to lymphocyte ratio (PLR). ${ }^{14,15}$ NLR in specific has been thoroughly investigated in other cancers and its predictive importance has been reported in cases such as colorectal cancer and colorectal liver metastases. ${ }^{16-18}$ Proposed mechanisms for the role of NLR include relative lymphocytopenia resulting in a poorer im- mune response to malignancy and a high neutrophil count enhancing the development of a neoplasmatic tumor through the release of vascular endothelial growth factor. ${ }^{16-18}$ Similarly PLR has been reported to hold a significant predictive value in regards to overall survival (OS) in ovarian cancer, colorectal cancer and also colorectal liver metastases. $^{19-22}$

In regards to borderline and locally advanced pancreatic cancer, Lee et al. ${ }^{23}$ from Seoul's Yonsei group reported recently NLR and PLR as useful markers of prediction for prognosis of advanced pancreatic cancers. Their patients' data included borderline or locally advanced pancreatic cancer patients receiving chemoradiotherapy as neoadjuvant or as definitive treatment. They showed that a higher NLR or PLR to be related to poor OS and progression free survival (PFS). Furthermore they reported that when both NLR and PLR values were high, they detected worse results for OS and PFS.

Previously Hasegawa et al. ${ }^{24}$ had investigated a cohort of pancreatic cancer patients receiving neoadjuvant chemoradiotherapy prior to complete surgical resection, and reported that a pre treatment NLR was an independent predictive marker of the pathological response to preoperative therapy. Asari et al. ${ }^{25}$ also reported, in a cohort of borderline pancreatic cancer (BR-PDAC) patients undergoing curative resection upfront, a preoperative NLR $>3$ to be an independent predictive risk factor. The authors also showed that elevated preoperative NLR and PLR values had a prognostic role regarding overall survival in BRPDAC patients.

\section{CHEMOTHERAPY}

Neoadjuvant treatment has been increasingly applied in patients with borderline resectable pancreatic cancer, as it ensures the delivery of multidisciplinary management to all patients who are about to undergo surgery, downstages tumors to facilitate surgical resection and also increases total and R0 resection rates. ${ }^{26,27}$ Current clinical trials and meta-analyses have been comparing the efficacy of the main cytotoxic agents used in neoadjuvant chemotherapy, ${ }^{26}$ as well as the role of radiotherapy (RT), currently used as chemoradiotherapy (cXRT) in the management of borderline resectable pancreatic cancer. ${ }^{28}$

Dhir et al. ${ }^{29}$ completed a systematic meta-analysis on the 
effects of neoadjuvant therapy in the treatment of nonmetastatic pancreatic cancer. Review was performed for 279 articles, but only 96 studies including 82 original articles and 14 abstracts, with a total of 5,520 patients, were selected for quantitative synthesis. Among these studies, 5 were a phase I, 3 were a phase I/II, 22 were a phase II and 1 was a phase III. Most of the studies were retrospective, while 11 studies were prospective. 1,014 patients with borderline resectable and locally advanced tumors were included in these studies, according to the criteria for resectability defined by an expert consensus statement in 2009. Chemotherapy alone was used as neoadjuvant therapy in $20(20.8 \%)$ studies, chemoradiotherapy in 33 (34.4\%) studies, chemotherapy with chemoradiation was used in $41(42.7 \%)$, and radiation alone was used in 2 (2.1\%) studies. 48 studies utilized the RECIST criteria for radiologic response to neoadjuvant therapy, while in 41 studies the criteria used were not adequately mentioned. Utilizing FOLFIRINOX as chemotherapy drug provided the best median survival of 22.1 months (range 16.7-34 months) followed by GTX (gemcitabine, taxane, capecitabine) at 19.4 months (range 15.6-25 months). Pooled median overall survival with single-agent chemotherapy (gemcitabine/5FU/capecitabine) was 14.7 months (range 9.1-47 months), in contrast to 16.1 months (range 7.3-45 months) when two drug regiments were used. Nevertheless, the above study failed to compare the efficacy of pre-operative chemotherapy agents and determine the role of radiation therapy and Contant $\mathrm{X}$ Ray Treatment (cXRT) specifically in patients with borderline resectable pancreatic cancer.

Gillen et al. ${ }^{30}$ published a second systematic review and meta-analysis of 111 retrospective and prospective studies from 1996 to 2009 concerning the effects of neoadjuvant therapy on tumor response and resection in pancreatic cancer. From 515 initially retrieved studies, 111 trials were included with a total of 4.394 patients. There were 15 phase I, 13 phase I/II, 28 phase II, 14 cohort studies and 41 case series. Trials were subdivided into groups considering initially resectable and non-resectable (borderline resectable/unresectable) tumors. Chemotherapy was applied as neoadjuvant treatment in 107 studies, utilizing gemcitabine, 5-FU, mitomycin $\mathrm{C}$, and platinum compounds as the main agents. Antibodies or tyrosine kinase inhibitors were also included in five studies. Radiotherapy was given in 104 studies with doses ranging from 24 Gy to $63 \mathrm{~Gy}$. Complete and partial responses were $2.2 \%$ (95\% CI: $1.3-3.3 \%$ ) and $25.8 \%$ (95\% CI: $20.2-31.8 \%$ ) respectively for patients treated with mono-chemotherapy, versus $5.3 \%$ (95\% CI: $3.8-7 \%$ ) and $34.7 \%$ (95\% CI: $28.9-40.9 \%$ ) respectively for combination therapy. Finally, analysis of trials with monotherapy versus poly-chemotherapy revealed an estimated $20 \%$ increase in the resection rates for patients with locally advanced - unresectable tumors, when combination chemotherapy was used. Data regarding the optimal cytotoxic and radiotherapeutic agents have not been mentioned on the above meta-analysis, and as a result further clinical trials are needed to determine the best regimen for neoadjuvant treatment.

Gemcitabine mono-therapy seems to be utilized in resectable tumors, as well as in the palliative treatment, whereas combination chemotherapy is administered in advanced diseases, as it prevails from gemcitabine -mono therapy. ${ }^{27}$ A phase III multicenter randomized ESPAC trial has compared the efficacy of gemcitabine alone or in combination with capecitabine in advanced pancreatic cancer. The study revealed that the addition of capecitabine in the neoadjuvant therapy with gemcitabine prolonged overall median survival from 19 to 26 months. Notably, induction chemotherapy followed by cXRT has been historically preferred for the treatment of borderline resectable pancreatic cancer, because of the lack of a robust response to chemotherapy alone; cXRT decreases the risk of local recurrence after polychemotherapy. ${ }^{26,31}$ In a small series published from Patel et al. ${ }^{32}$ in 2011, 18 borderline resectable pancreatic cancer patients received induction chemotherapy with GTX (gemcitabine, docetaxel, capecitabine) followed by 5-FU Intensity-modified Radiation Therapy (IMRT). Out of the 17 patients who completed neoadjuvant treatment, 14 underwent surgery and 8 successfully achieved a Whipple operation with R0 resection margins. Progression-free survival of all patients was 10.48 months and median overall survival was 15.6 months, thus succeeding in clinical outcomes similar to resectable pancreatic cancer. ${ }^{32}$ However, a recent study of 575 patients with locally advanced pancreatic cancer revealed that FOLFIRINOX chemotherapy resulted in significantly higher secondary resection rates and overall survival compared to gemcitabine-based cXRT. ${ }^{33}$ Resection 
rates were $60.8 \%$ in the FOLFIRINOX group, in contrast with $48 \%$ after gemcitabine and radiation. Three- year survival rate also seemed to be higher in FOLFIRINOX group than any other treatment group $(28.1 \%$ versus $23.2 \%) .{ }^{33}$ Furthermore, in a clinical trial published from Boone et al., ${ }^{34} 12$ patients with borderline resectable and 13 with unresectable pancreatic cancer underwent treatment with FOLFIINOX receiving a mean of 5 cycles. Overall negative resection rate was $33 \%$; with $55 \%$ of borderline resectable and $10 \%$ of unresectable patients achieving R0 resection margins. ${ }^{34}$ FOLFIRINOX was also found to be more cytotoxic agent compared with gemcitabine for patients with metastatic pancreatic tumors. ${ }^{35}$ Conroy et al. ${ }^{35}$ reported a study of 342 metastatic patients, treated with FOLFIRINOX or gemcitabine for six months. Pooled estimates showed that the response rate in the FOLFIRINOX group was 31.6\% (95\% CI: 24.7-39.1\%) versus $9.4 \%$ (95\% CI: 5.4-14.7\%) in patients treated with gemcitabine. The median overall survival for patients treated with FOLFIRINOX and gemcitabine was 11.1 months (95\% CI: 9-13.1) and 6.8 months (95\% CI: 5.57.6) respectively. Median progression - free survival was also higher in the FOLFIRINOX group; with 6.4 months in contrary to 3.3 months in the gemcitabine group.

Besides FOLFIRINOX, gemcitabine/nab-paclitaxel/S-1 (GAS) has also administered as combination neoadjuvant treatment in patients with borderline resectable pancreatic cancer. ${ }^{27}$ A phase I study of the above mentioned chemo- therapy agents has been published from Kondo et al. ${ }^{36}$ in December 2016 including a total of 16 patients with borderline resectable disease. Complete response was not achieved in any patient; 5 patients had partial response, 10 had stable disease, while one patient was found with progressive disease after having completed five cycles of chemotherapy. From 13 of 16 patients who underwent surgery with curative intent, R0 resection was performed in 12 of them. The initial results of the study indicated that GASchemotherapy was efficient without severe toxicity. ${ }^{36}$ Because of the high response and resection rates of FOLFIRINOX and GAS chemotherapy in the neoadjuvant treatment of pancreatic cancer, these protocols have gained increasing interest particularly for borderline and unresectable cancer during recent years. ${ }^{31}$

Ongoing clinical trials are already conducted to confirm the best neoadjuvant treatment protocol for patients with borderline and locally advanced pancreatic cancer. ${ }^{26}$ In June 2018 Conroy et al. ${ }^{37}$ from ASCO university presented a randomized phase III trial of 493 patients with resectable pancreatic ductal adenocarcinoma treated with mFOLFIRINOX after undergoing surgery. In contrary to gemcitabine alone, adjuvant treatment with mFOLFIRINOX, a modified agent including oxaliplatin, leucovorin, irinotecan and fluorouracil, augmented the median disease-free survival (21.6 versus 12.8 months), as well as the median overall survival (54.4 versus 35 months), with manageable heamatologic side effects. ${ }^{37}$ Observing the effectiveness of

Table 1. Median overall survival and resection rates after utilizing chemotherapy as treatment modality

\begin{tabular}{|c|c|c|c|}
\hline Chemotherapy & & $\begin{array}{l}\text { Median overall } \\
\text { survival (months) }\end{array}$ & $\begin{array}{l}\text { Resection } \\
\text { rate }(\%)\end{array}$ \\
\hline \multicolumn{4}{|l|}{ Folfirinox } \\
\hline Dhir et al. ${ }^{29} 2017$ & FOLFIRINOX & 22.1 & - \\
\hline Nitsche et al. ${ }^{59} 2015$ & FOLFIRINOX & 31 & 29 \\
\hline Paniccia et al. $^{60} 2014$ & FOLFIRINOX & - & 85 \\
\hline Boone et al. ${ }^{34} 2013$ & FOLFIRINOX & - & 43 \\
\hline Christians et al. ${ }^{61} 2014$ & FOLFIRINOX & - & 67 \\
\hline Tinchon et al. ${ }^{62} 2013$ & FOLFIRINOX & - & 83 \\
\hline Hosein et al. ${ }^{63} 2012$ & FOLFIRINOX & - & 55 \\
\hline Hackert et al. ${ }^{33} 2016$ & FOLFIRINOX & 16 & 60.8 \\
\hline Conroy et al. $^{35} 2011$ & FOLFIRINOX & 11.1 & - \\
\hline \multicolumn{4}{|l|}{ Other } \\
\hline Dhir et al. $^{29} 2017$ & GTX (gemcitabine, taxane, capecitabine) & 19.4 & - \\
\hline Gillen $^{30} 2010$ & Combination chemotherapy & - & 33 \\
\hline Hackert et al. ${ }^{33} 2016$ & Gemcitabine-based cXRT & 16.5 & 48 \\
\hline Conroy et al. ${ }^{35} 2011$ & Gemcitabine & 6.8 & - \\
\hline Kondo et al. ${ }^{36} 2017$ & GAS (gemcitabine/nab-paclitaxel/S-1) & - & 81.25 \\
\hline
\end{tabular}


mFOLFIRINOX in adjuvant treatment, Dr. Conroy indicated that it could be also successfully utilized as a neoadjuvant therapy regiment for patients with metastatic pancreatic cancer and appear more effective than gemcitabine, which is the current standard of treatment (Table $1) .^{37}$

\section{RADIATION THERAPY}

Although novel agents, such as FOLFIRINOX and nab-paclitaxel, have been added to neoadjuvant treatment sequencing for pancreatic cancer, challenges still exist in improving survival outcomes and disease local control. ${ }^{38}$ The LAP07 randomized clinical trial proved that the addition of conventionally chemoradiotherapy to chemotherapy did not improve the overall survival in locally advanced pancreatic cancer or reduce treatment toxicity. ${ }^{39}$ In a recent meta-analysis of 41 prospective studies, Chang et al. ${ }^{40}$ also evaluated the role of consolidation chemoradiotherapy (CCRT) followed by chemotherapy in locally advanced pancreatic cancer. CCRT utilized in a total of 1018 patients, while chemotherapy alone in 954 patients. No significant difference was noted between 1-year survival benefit with the administration of CCRT after induction chemotherapy in comparison to chemotherapy alone. $^{40}$ As a result, current studies aim to assess the benefit of different techniques and modalities of radiotherapy in combination with systemic therapies. ${ }^{38,41}$ A randomized phase II ESPAC-5F trial is already about to assess the effectiveness and safety of chemoradiotherapy with gemcitabine or capecitabine following gemcitabine plus capecitabine chemotherapy in patients with locally advanced pancreatic cancer. If encouraging, this trial will determine the experimental arm of a phase III study comparing radiation therapy against chemotherapy alone. Stereotactic body radiation therapy (SBRT) is an emerging approach which enables the delivery of higher doses of radiation to the tumor, with minimal doses to the adjacent normal tissues. ${ }^{38}$ In contrast with conventionally fractionated radiotherapy (CFRT), where the duration of systemic treatment is up to 6 weeks, patients treated with SBRT need to be off systemic therapy for a short period of time, typically one to two weeks, as it employs a hypofractionated regiment. ${ }^{41}$ In a retrospective review of 8,450 patients with locally advanced pancreatic cancer using the National
Cancer Database, receipt of SBRT leaded to higher rates of median overall survival (13.9 months vs 11.6 months) and 2 -year overall survival $(21.7 \%$ vs $16.5 \%)$ in comparison with CFRT. ${ }^{42}$ Furthermore, pooled results from current literature proved that tumor local control was almost $80 \%$ after one year treatment with SBRT. ${ }^{41}$ Gastrointestinal toxicity rates caused by higher doses of SBRT could be eliminated with the utilization of stereotactic MRIguided radiotherapy (SMART), allowing SBRT to achieve high doses of hypofractionated radiation without damaging surrounding radiosensitive normal structures. ${ }^{38}$

Intensity-modulated radiation therapy (IMRT) is also an irradiation therapy which achieves an increase in target tumor dose, while minimizing toxicity to surrounding normal tissues. ${ }^{43}$ Masui et al. ${ }^{43}$ investigated the advantages of IMRT over conventional RT for patients with borderline resectable pancreatic cancer with arterial abutment. A total of 29 patients were enrolled in the study; 18 of them underwent IMRT, while 12 of them conventional RT before resection. R0 resection rates were $84 \%$ for the patients treated with IMRT and $83 \%$ for the RT group. Local control rate, according to the Evans classification, was higher in the IMRT group than in the RT group, while median overall survival was 32 months and 13.8 months respectively. ${ }^{43}$ Several recent studies have proved that the utilization of IMRT in locally advanced pancreatic cancer leaded to excellent local control and survival rates. ${ }^{44}$ In addition, Goto et al. ${ }^{44}$ implemented dynamic tumor-tracking IMRT (DTT-IMRT) with real-time monitoring, using a gimbal mounted linac, in patients with locally advanced pancreatic tumors, so as to manage tumor motion and avoid deviation between planned and actual dose distribution. In a follow-up period of 22.9 months, median overall survival was estimated at 23.6 months and locoregional progression free survival rates were $90.9 \%$ and $37.9 \%$ after one and two years respectively, while severe gastrointestinal toxicity was noted in only one patient, compared to the outcomes of the previously mentioned LAP07 clinical trial, where the median survival was 15.2 months and grade 3-4 toxicity rates were $23.1 \%$. ${ }^{44,39}$

Last but not least, particle therapy, including proton and carbon ion therapy, is being explored as a novel treatment modality for pancreatic cancer, providing greater biological effectiveness because of a higher linear energy transfer compared to photon treatment, thereby decreasing 
Table 2. Median overall survival and resection rates after utilizing radiotherapy as treatment modality

\begin{tabular}{|c|c|c|c|}
\hline Radiation therapy & & $\begin{array}{c}\text { Median overall } \\
\text { survival (months) }\end{array}$ & Resection rate $(\%)$ \\
\hline Masui et al. ${ }^{43} 2017$ & Intensity-modified Radiation Therapy (IMRT) & 32 & 84 \\
\hline Masui et al. ${ }^{43} 2017$ & Conventionally fractionated radiotherapy (CFRT) & 13.8 & 83 \\
\hline Goto et al. $^{44} 2018$ & DTT-IMRT & 23.6 & - \\
\hline Patel et al. 2011 & IMRT in combination with GTX & 15.6 & 47 \\
\hline Zhong et al. $^{42} 2017$ & Stereotactic body radiation therapy (SBRT) & 13.9 & 10.8 \\
\hline Zhong et al. ${ }^{42} 2017$ & Conventionally fractionated radiotherapy (CFRT) & 11.6 & 9.2 \\
\hline Kawashiro et al. ${ }^{46} 2018$ & Carbon ion therapy in combination with gemcitabine & 21.5 & - \\
\hline
\end{tabular}

dose to adjacent tissues within the exit beam path. ${ }^{41}$ In a dosimetric trial comparing particle therapy and IMRT, 13 patients with unresectable cancer of the pancreatic head were treated with proton plans calculated to $55 \mathrm{~Gy}$ in 25 fractions. ${ }^{45}$ Proton therapy minimized stomach, duodenum and small bowel dose in the mid to low dose regions compared to IMRT, but it failed to derive reduced dose in the mid to high dose regions, as well. ${ }^{45}$ Further follow-up is needed to define the biological significance of decreased dose to the low dose regions. In another multi-institutional study, 72 patients with locally advanced pancreatic cancer were treated with carbon ion therapy in combination with precedent or concurrent gemcitabine. ${ }^{46}$ Median overall survival was 21.5 months (95\% CI: 11.8 31.2 months), while concurrent chemotherapy and the higher prescribed dose of radiation were statistically significant factors regarding overall survival. ${ }^{46}$ In total, new radiotherapy modalities are an encouraging approach for the treatment of non-resectable pancreatic cancer, alone or in combination with systemic chemotherapy (Table 2).

\section{IMMUNOTHERAPIES IN PANCREATIC CANCER}

Immunotherapy against cancers has contributed to encouraging outcomes in preclinical models over decades, thus recent clinical trials have investigated its therapeutic application in counteracting pancreatic cancer cells. ${ }^{47}$ Programmed cell death receptor ligand (PD1/PDL1)- inhibitors seem to be a promising therapeutic approach in a number of human cancers, as well as in pancreatic cancer, enhancing the anti-tumor immune response in PDL1upregulated tumors in preclinical models. ${ }^{48}$ Birnbaum et al. ${ }^{48}$ analyzed PDL1 mRNA expression in 453 pancreatic can- cer samples and demonstrated that $P D L 1$ upregulation was observed in $19 \%$ of cancer samples, compared to normal samples, leading to shorter disease-free survival and overall survival rates. In a multicenter phase I trial, a total of 207 patients with selected advanced cancers, received intravenous anti-PDL1 antibody. ${ }^{49}$ Response rates were ranged from $6 \%$ to $17 \%$ of all patients and disease stabilization was extended in patients with advanced cancers, including non-small-cell cancer, melanoma, renal-cell cancer and ovarian cancer, but not in patients with pancreatic cancer. ${ }^{49}$ Additional clinical trials are needed to evaluate efficacy and safety of PDL1 inhibitors specifically in patients with advanced pancreatic cancer.

CTLA-4 (CD152) is another checkpoint receptor target for immunotherapy in pancreatic cancer, which controls early stages of T-cell activation. ${ }^{47}$ Preclinical models presented improved tumor local control and its decrease after utilization of the anti-CTLA-4 agent, ipilimumab. ${ }^{47}$ Le et al. ${ }^{50}$ demonstrated a phase $\mathrm{Ib}$ clinical trial to evaluate safety, survival rates and $\mathrm{T}$-cell responses to ipilimumab alone (arm 1) and in combination with GM-CSF vaccine (arm 2) in 30 patients with previously treated pancreatic cancer. The combination of drugs resulted in prolonged disease stabilization and improved median and 1-year overall survival; 3.6 vs 5.7 months and 7 vs $27 \%$, respectively. ${ }^{50}$ Investigations of Washington University presented that CSF1R blockade also upregulated PDL1 and CTLA4, and as a result, combining these agents with CSF1R blockade robustly provoked tumor regressions. ${ }^{51}$ These findings provide a reasoning to reprogram immunosuppressive myeloid cell populations in the tumor microenvironment under circumstances that can significantly strengthen the treatment outcomes of checkpoint-based immunotherapeutics (Table 3). ${ }^{51}$ 
Table 3. Immunotherapies in pancreatic cancer

\begin{tabular}{llr}
\multicolumn{1}{c}{ Immunotherapies } & & $\begin{array}{c}\text { Median overall } \\
\text { survival (months) }\end{array}$ \\
\hline Mahalingam et al. ${ }^{13} 2018$ & Reolysin in combination with gemcitabine & 10.2 \\
Le et al. ${ }^{50} 2013$ & Ipilimumab (anti-CTLA-4 agent) in combination with GM-CSF vaccine & 5.7 \\
Le et al. ${ }^{50} 2013$ & Ipilimumab & 3.6 \\
\hline
\end{tabular}

\section{VASCULAR RESECTIONS}

Historically, arterial abutment has been considered as a contraindication to pancreatectomy (PD), due to increased perioperative morbidity and mortality specially related to arterial resection and reconstruction, compared to standard PD. ${ }^{1}$ Concurrent PD with vascular resection applies to increase the possibility for negative resection margins. ${ }^{1}$ Celiac axis or common hepatic artery (CHA) resections are carried out more often, whereas resection of the superior mesenteric artery (SMA) is scarcely recommendable, even if it can be technically possible. ${ }^{1}$ Lately, with the incorporation of neoadjuvant therapy, removement of the primary tumor is feasible, even in the setting of complex encasement or occlusion of SMA. ${ }^{1}$

Vicente et al. ${ }^{52}$ presented a series of 25 cases of locally advanced pancreatic cancer, 12 of them undergoing PD with resection of SMA and superior mesenteric vein (SMV) after receiving neoadjuvant treatment. Concominant SMA and SMV resection was applied to five patients, while post-operative mortality occurred in only one of them. Gemcitabine plus nab-paclitaxel was administered in one patient, whose disease-free survival was 31 months from surgery. ${ }^{52}$ Moreover, an ongoing multicenter phase II clinical trial (JASPAC05) is about to evaluate neoadjuvant S-1 with concurrent radiotherapy as preoperative treatment for patients with borderline resectable pancreatic tumors occluding major vessels. ${ }^{53}$ The primary objective of the study is $\mathrm{R} 0$ resection rate, while the secondary endpoints include safety of systematic treatment and subsequent surgery, survival rates, response rates to treatment and pathological response rate. ${ }^{53}$ Fujii et al. ${ }^{54}$ published another study, comparing the use of neoadjuvant chemoradiotherapy (NACRT) versus upfront surgery in patients with borderline resectable tumors, with abutment of SMA or CHA. 21 patients were treated with neoadjuvant therapy followed by surgery with the rationale of preserving the artery, while 71 patients underwent surgery first. Eventually, 18 and 50 patients from each group underwent resection, respectively. A positive microscopic resection margin was observed in $60 \%$ of the patients who underwent upfront surgery, whereas the total number of patients who received systemic therapy before surgery achieved an R0 tumor resection. Positive lymph nodes metastases were significantly lower in the NACRT group, as well. In addition, the estimated 1- and 2-year survival rates were higher in the NACRT group than in the upfront surgery group, among the matched patients. ${ }^{54}$ Consequently, neoadjuvant therapy followed by surgery leaded to greater clinical advantages in patients with pancreatic tumors involving major arteries. Overall, PD for non-resectable pancreatic tumors affecting major arteries still remains a challenging approach with high mortality rates. Nevertheless, in selected cases, such as young age and high radiological and biological response to systemic therapy, combined arterial resection with PD should be taken into account as the best treatment option resulting in potential locoregional control and increased survival rates. ${ }^{52,54}$

Generally, thanks to novel perioperative management techniques and multidisciplinary systemic treatment, concomitant venous and arterial resection and reconstruction have thus been aggressively attempted in combination with significantly higher negative resection margins and disease-free survival rates versus upfront vascular resection and adjuvant chemoradiation therapy. ${ }^{1}$ Ielpo et al. ${ }^{55}$ reported a study of 81 patients with potentially resectable pancreatic cancer comparing neoadjuvant treatment with gemcitabine plus nab-paclitaxel versus upfront surgery. Forty-five patients with borderline resectable tumors with abutment of major vessels were included in the study, according to NCCN guidelines. A total of 26 patients out of 45 completed neoadjuvant treatment and 16 patients (61.5\%) underwent PD, while 19 patients out of 45 underwent upfront surgical excision; neoadjuvant treatment resulted in four times higher overall survival (43.6 vs 13.5 months) compared to surgery first. ${ }^{55}$ Although vascular re- 
section following NACRT seems to be a more technically challenging procedure in comparison with a surgery-first approach, due to the presence of scar tissue or insistence of tumor-vessel abutment after NACRT, this approach leads to significantly higher $\mathrm{R} 0$ resection and median overall survival rates. ${ }^{56}$ Even if apparent improved tumorvessel interactions are rarely noticed on post-treatment CT scan, patients with adequate performance status and without metastatic disease should be candidates for surgery. ${ }^{56}$

\section{DISCUSSION}

The contribution of neoadjuvant treatment for borderline or locally advanced pancreatic cancer is well recognized for both patients and physicians, as it increases negative resection margins and progression-free survival andfacilitates the technical aspects of surgical resection, concerning the relationship of the tumor to surrounding vascular structures. ${ }^{26,57}$ A recent meta-analysis investigated the radiologic response, resection rates and overall survival in 5520 patients with pancreatic cancer. ${ }^{29}$ Pooled estimates of patients with borderline resectable/locally advanced tumors showed a complete response in $0 \%$, partial response in $20 \%$, stable disease in $59 \%$ and progression in $16 \%$ during therapy. Resections were performed in 53\% (95\% CI: $36-70 \%$ ) and R0 resection rates were 39\% (95\% CI: $27-52 \%$ ) for borderline resectable/locally advanced patients on an intention to-treat-basis with a median survival of 26 months (range 13-47.4) and a progression- free survival of 12.5 months (range 9-19.7). Finally, the R0 resection rates were $85 \%$ in patients who underwent resection. ${ }^{29}$ Furthermore, Gillen et al. ${ }^{30}$ showed that in non- resectable tumor patients complete response were $4.8 \%$ (95\% CI: $3.5-6.4 \%)$, partial responses were $30.2 \%$ (95\% CI: $24.5-36.3 \%$ ), whereas stable disease was observed in $41.6 \%$ (95\% CI: 34.6-48.7\%) and progression in $20.8 \%$ (95\% CI: $14.5-27.8 \%)$. Resectability was estimated to $33.2 \%$ (95\% CI: $25.8-41.1 \%$ ) of initially staged non-resectable tumor patients and it was more frequent in the group of patients who received combination chemotherapy with 33\%. Additionally, R0 resection margins were observed in $79.2 \%$ (95\% CI: $72.4-85.2 \%$ ), which were comparable to the group of initially resectable tumor patients $(82.1 \%$, 95\% CI: 73.1-89.6\%). The estimated median survival for the initially non- resectable patients who underwent resection was 20.5 (range 9-62) months, in comparison to 10.2 (range 6.21) months of patients who did not undergo resection after pretreatment. ${ }^{30}$

The most significant finding of these two meta-analyses was that approximately one third of patients primarily staged as non-resectable tumors could be successfully resected after neoadjuvant treatment with an estimated median survival within the spectrum of primarily resectable tumor patients. In 2015, Zhan et al. ${ }^{27}$ also performed another systematic review and meta-analysis of prospective studies on neoadjuvant therapy in pancreatic cancer. Thirty-nine studies were included with a total of 1,458 patients covering a period of 15 years (2000 to 2015). Studies were divided into three groups based on the AHPBA/NCCN standard, including 592 patients with borderline and/or locally advanced pancreatic cancer. Their analysis demonstrated that neoadjuvant treatment had been proven to be beneficial for patients with borderline or locally advanced tumors, as it provided a better median survival of 21.8 months, which was within the range of patients with primary resection. Moreover, the resection rate for patients with borderline resectable and locally advanced disease was 40.2\% (95\% CI: 28.3-53.4\%), whereas margin- negative (R0) resections were achieved in $79.4 \%$ of the resected patients. ${ }^{27}$ In September 2011 Assifi et al. ${ }^{58}$ also performed another systematic review and metaanalysis of 14 prospective phase II trials, including 536 patients with initially resectable, borderline or unresectable pancreatic tumors, investigating the effects of neoadjuvant therapy. Local recurrence was found to be $11.1 \%$ (95\% CI: 5.6-18\%) in all patients according to data reported from eight trials, while distant recurrence was analyzed in nine trials and it was estimated as $43.9 \%(95 \%$ CI: $34.5-53.6 \%$ ) in all patients, irrespectively of being initially resectable or not. Complete response was only $1.8 \%$ (95\% CI: 0.6-3.6\%) for all patients. Partial response was significantly higher for patients with advanced disease (31.8\%, 95\% CI: 24.2-39.8\%), in contrast to resectable tumor patients $(9.5 \%, 95 \%$ CI: $2.9-19.4 \%)$. Stable disease was $40.9 \%$ (95\% CI: 32.8-49.3\%) in patients with borderline/unresectable tumors, compared to $73.9 \%$ (95\% CI: 63.2-83.3) in patients with resectable tumors. Progressive disease was $18.9 \%$ in all patients. Resection rates were $31.6 \%$ (95\% CI: $14-52.5 \%)$ in patients with borderline/ unresectable disease. Finally, the overall median survival 
has not found to be significantly different between resectable and advanced tumor patients. ${ }^{58}$ Consequently, nearly one-third of tumors initially deemed marginal for operative intervention were eventually able to be resected following neoadjuvant treatment.

\section{REFERENCES}

1. Luketina RR, Hackert T, Büchler MW. Vascular resection in pancreatic cancer. Indian J Surg 2015;77:381-386.

2. Tamburrino D, Partelli S, Crippa S, Manzoni A, Maurizi A, Falconi M. Selection criteria in resectable pancreatic cancer: a biological and morphological approach. World J Gastroenterol 2014;20:11210-11215.

3. Tempero MA, Malafa MP, Al-Hawary M, Asbun H, Bain A, Behrman SW, et al. Pancreatic adenocarcinoma, version 2.2017, NCCN clinical practice guidelines in oncology. J Natl Compr Canc Netw 2017;15:1028-1061.

4. Fokas E, O'Neill E, Gordon-Weeks A, Mukherjee S, McKenna WG, Muschel RJ. Pancreatic ductal adenocarcinoma: from genetics to biology to radiobiology to oncoimmunology and all the way back to the clinic. Biochim Biophys Acta 2015;1855:61-82.

5. Kretz AL, von Karstedt S, Hillenbrand A, Henne-Bruns D, Knippschild U, Trauzold A, et al. Should we keep walking along the trail for pancreatic cancer treatment? Revisiting TNF-related apoptosis-inducing ligand for anticancer therapy. Cancers (Basel) 2018;10. pii:E77.

6. Kamil F, Rowe JH. How does the tumor microenvironment play a role in hepatobiliary tumors? J Gastrointest Oncol 2018;9:180195.

7. Melisi D, Garcia-Carbonero R, Macarulla T, Pezet D, Deplanque G, Fuchs M, et al. A phase II, double-blind study of galunisertib+gemcitabine (GG) vs gemcitabine+placebo (GP) in patients (pts) with unresectable pancreatic cancer (PC). J Clin Oncol 2016;34:4019.

8. Kindler HL, Ioka T, Richel DJ, Bennouna J, Létourneau R, Okusaka T, et al. Axitinib plus gemcitabine versus placebo plus gemcitabine in patients with advanced pancreatic adenocarcinoma: a double-blind randomised phase 3 study. Lancet Oncol 2011;12:256-262.

9. Ansari D, Friess H, Bauden M, Samnegård J, Andersson R. Pancreatic cancer: disease dynamics, tumor biology and the role of the microenvironment. Oncotarget 2018;9:6644-6651.

10. Carapuça EF, Gemenetzidis E, Feig C, Bapiro TE, Williams MD, Wilson AS, et al. Anti-stromal treatment together with chemotherapy targets multiple signalling pathways in pancreatic adenocarcinoma. J Pathol 2016;239:286-296.

11. Duluc C, Moatassim-Billah S, Chalabi-Dchar M, Perraud A, Samain R, Breibach F, et al. Pharmacological targeting of the protein synthesis mTOR/4E-BP1 pathway in cancer-associated fibroblasts abrogates pancreatic tumour chemoresistance. EMBO Mol Med 2015;7:735-753.

12. Guo Q, Jin Z, Yuan Y, Liu R, Xu T, Wei H, et al. New mechanisms of tumor-associated macrophages on promoting tumor progression: recent research advances and potential targets for tumor immunotherapy. J Immunol Res 2016;2016:9720912.

13. Mahalingam D, Goel S, Aparo S, Patel Arora S, Noronha N, Tran H, et al. A phase II study of pelareorep (REOLYSIN(®)) in combination with gemcitabine for patients with advanced pancreatic adenocarcinoma. Cancers (Basel) 2018;10. pii:E160.

14. Proctor MJ, Morrison DS, Talwar D, Balmer SM, Fletcher CD,
O'Reilly DS, et al. A comparison of inflammation-based prognostic scores in patients with cancer. A glasgow inflammation outcome study. Eur J Cancer 2011;47:2633-2641.

15. Kwon HC, Kim SH, Oh SY, Lee S, Lee JH, Choi HJ, et al. Clinical significance of preoperative neutrophil-lymphocyte versus platelet-lymphocyte ratio in patients with operable colorectal cancer. Biomarkers 2012;17:216-222.

16. Halazun KJ, Aldoori A, Malik HZ, Al-Mukhtar A, Prasad KR, Toogood GJ, et al. Elevated preoperative neutrophil to lymphocyte ratio predicts survival following hepatic resection for colorectal liver metastases. Eur J Surg Oncol 2008;34:55-60.

17. Malietzis G, Giacometti M, Askari A, Nachiappan S, Kennedy $\mathrm{RH}$, Faiz OD, et al. A preoperative neutrophil to lymphocyte ratio of 3 predicts disease-free survival after curative elective colorectal cancer surgery. Ann Surg 2014;260:287-292.

18. Giakoustidis A, Neofytou K, Khan AZ, Mudan S. Neutrophil to lymphocyte ratio predicts pattern of recurrence in patients undergoing liver resection for colorectal liver metastasis and thus the overall survival. J Surg Oncol 2015;111:445-450.

19. Szkandera J, Pichler M, Absenger G, Stotz M, Arminger F, Weissmueller $\mathrm{M}$, et al. The elevated preoperative platelet to lymphocyte ratio predicts decreased time to recurrence in colon cancer patients. Am J Surg 2014;208:210-214.

20. Asher V, Lee J, Innamaa A, Bali A. Preoperative platelet lymphocyte ratio as an independent prognostic marker in ovarian cancer. Clin Transl Oncol 2011;13:499-503.

21. Liu H, DU X, Sun P, Xiao C, Xu Y, Li R. [Preoperative platelet-lymphocyte ratio is an independent prognostic factor for resectable colorectal cancer]. J Shouth Med Univ 2013;33:70-73. Chinese.

22. Neofytou K, Smyth EC, Giakoustidis A, Khan AZ, Cunningham D, Mudan S. Elevated platelet to lymphocyte ratio predicts poor prognosis after hepatectomy for liver-only colorectal metastases, and it is superior to neutrophil to lymphocyte ratio as an adverse prognostic factor. Med Oncol 2014;31:239.

23. Lee BM, Chung SY, Chang JS, Lee KJ, Seong J. The neutrophil-lymphocyte ratio and platelet-lymphocyte ratio are prognostic factors in patients with locally advanced pancreatic cancer treated with chemoradiotherapy. Gut Liver 2018;12:342-352.

24. Hasegawa S, Eguchi H, Tomokuni A, Tomimaru Y, Asaoka T, Wada $\mathrm{H}$, et al. Pre-treatment neutrophil to lymphocyte ratio as a predictive marker for pathological response to preoperative chemoradiotherapy in pancreatic cancer. Oncol Lett 2016;11: 1560-1566.

25. Asari S, Matsumoto I, Toyama H, Shinzeki M, Goto T, Ishida $\mathrm{J}$, et al. Preoperative independent prognostic factors in patients with borderline resectable pancreatic ductal adenocarcinoma following curative resection: the neutrophil-lymphocyte and platelet-lymphocyte ratios. Surg Today 2016;46:583-592.

26. Christians KK, Heimler JW, George B, Ritch PS, Erickson BA, Johnston F, et al. Survival of patients with resectable pancreatic cancer who received neoadjuvant therapy. Surgery 2016;159: 893-900.

27. Zhan HX, Xu JW, Wu D, Wu ZY, Wang L, Hu SY, et al. Neoadjuvant therapy in pancreatic cancer: a systematic review and meta-analysis of prospective studies. Cancer Med 2017;6: 1201-1219.

28. Brunner TB, Scott-Brown M. The role of radiotherapy in multimodal treatment of pancreatic carcinoma. Radiat Oncol 2010;5: 64.

29. Dhir M, Malhotra GK, Sohal DPS, Hein NA, Smith LM, O'Reilly EM, et al. Neoadjuvant treatment of pancreatic adenocarcinoma: a systematic review and meta-analysis of 5520 patients. World J Surg Oncol 2017;15:183. 
30. Gillen S, Schuster T, Meyer Zum Büschenfelde C, Friess H, Kleeff J. Preoperative/neoadjuvant therapy in pancreatic cancer: a systematic review and meta-analysis of response and resection percentages. PLoS Med 2010;7:e1000267.

31. Heinrich S, Lang $H$. Neoadjuvant therapy of pancreatic cancer: definitions and benefits. Int J Mol Sci 2017;18. pii:E1622.

32. Patel M, Hoffe S, Malafa M, Hodul P, Klapman J, Centeno B, et al. Neoadjuvant GTX chemotherapy and IMRT-based chemoradiation for borderline resectable pancreatic cancer. J Surg Oncol 2011;104:155-161.

33. Hackert T, Sachsenmaier M, Hinz U, Schneider L, Michalski CW, Springfeld C, et al. Locally advanced pancreatic cancer: neoadjuvant therapy with folfirinox results in resectability in $60 \%$ of the patients. Ann Surg 2016;264:457-463.

34. Boone BA, Steve J, Krasinskas AM, Zureikat AH, Lembersky $\mathrm{BC}$, Gibson MK, et al. Outcomes with FOLFIRINOX for borderline resectable and locally unresectable pancreatic cancer. J Surg Oncol 2013;108:236-241.

35. Conroy T, Desseigne F, Ychou M, Bouché O, Guimbaud R, Bécouarn Y, et al. FOLFIRINOX versus gemcitabine for metastatic pancreatic cancer. N Engl J Med 2011;364:1817-1825.

36. Kondo N, Murakami Y, Uemura K, Sudo T, Hashimoto Y, Nakagawa $\mathrm{N}$, et al. A phase 1 study of gemcitabine/nab-paclitaxel/S-1 (GAS) combination neoadjuvant chemotherapy for patients with locally advanced pancreatic adenocarcinoma. Cancer Chemother Pharmacol 2017;79:775-781.

37. Conroy T, Hammel P, Hebbar M, Abdelghani MB, Wei AC, Raoul JL, et al. Unicancer GI PRODIGE 24/CCTG PA.6 trial: a multicenter international randomized phase III trial of adjuvant mFOLFIRINOX versus gemcitabine (gem) in patients with resected pancreatic ductal adenocarcinomas. J Clin Oncol 2018. doi: 10.1200/JCO.2018.36.18_suppl.LBA4001.

38. Luterstein E, Cao M, Lamb J, Raldow AC, Low DA, Steinberg ML, et al. Stereotactic MRI-guided adaptive radiation therapy (SMART) for locally advanced pancreatic cancer: a promising approach. Cureus 2018;10:e2324.

39. Hammel P, Huguet F, van Laethem JL, Goldstein D, Glimelius B, Artru P, et al. Effect of chemoradiotherapy vs chemotherapy on survival in patients with locally advanced pancreatic cancer controlled after 4 months of gemcitabine with or without erlotinib: the LAP07 randomized clinical trial. JAMA 2016;315:18441853.

40. Chang JS, Chiu YF, Yu JC, Chen LT, Ch'ang HJ. The role of consolidation chemoradiotherapy in locally advanced pancreatic cancer receiving chemotherapy: an updated systematic review and meta-analysis. Cancer Res Treat 2018;50:562-574.

41. Ng SP, Herman JM. Stereotactic radiotherapy and particle therapy for pancreatic cancer. Cancers (Basel) 2018;10. pii:E75.

42. Zhong J, Patel K, Switchenko J, Cassidy RJ, Hall WA, Gillespie $\mathrm{T}$, et al. Outcomes for patients with locally advanced pancreatic adenocarcinoma treated with stereotactic body radiation therapy versus conventionally fractionated radiation. Cancer 2017;123: 3486-3493.

43. Masui T, Takaori K, Anazawa T, Sato A, Nakano K, Uchida $\mathrm{Y}$, et al. A prospective study of intensity-modified radiation therapy in comparison with conventional 3D-RT for BR pancreatic cancer patients with arterial involvement. Anticancer Res 2017; 37:7023-7030.

44. Goto Y, Ashida R, Nakamura A, Itasaka S, Shibuya K, Akimoto $\mathrm{M}$, et al. Clinical results of dynamic tumor tracking intensitymodulated radiotherapy with real-time monitoring for pancreatic cancers using a gimbal mounted linac. Oncotarget 2018;9:2362823635.

45. Thompson RF, Mayekar SU, Zhai H, Both S, Apisarnthanarax
S, Metz JM, et al. A dosimetric comparison of proton and photon therapy in unresectable cancers of the head of pancreas. Med Phys 2014;41:081711.

46. Kawashiro S, Yamada S, Okamoto M, Ohno T, Nakano T, Shinoto M, et al. Multi-institutional study of carbon-ion radiotherapy for locally advanced pancreatic cancer: Japan carbon-ion radiation oncology study group (J-CROS) study 1403 pancreas. Int J Radiat Oncol Biol Phys 2018;101:1212-1221.

47. Thind K, Padrnos LJ, Ramanathan RK, Borad MJ. Immunotherapy in pancreatic cancer treatment: a new frontier. Therap Adv Gastroenterol 2017;10:168-194.

48. Birnbaum DJ, Finetti P, Lopresti A, Gilabert M, Poizat F, Turrini $\mathrm{O}$, et al. Prognostic value of PDL1 expression in pancreatic cancer. Oncotarget 2016;7:71198-71210.

49. Brahmer JR, Tykodi SS, Chow LQ, Hwu WJ, Topalian SL, Hwu $\mathrm{P}$, et al. Safety and activity of anti-PD-L1 antibody in patients with advanced cancer. N Engl J Med 2012;366:2455-2465.

50. Le DT, Lutz E, Uram JN, Sugar EA, Onners B, Solt S, et al. Evaluation of ipilimumab in combination with allogeneic pancreatic tumor cells transfected with a GM-CSF gene in previously treated pancreatic cancer. J Immunother 2013;36:382-389.

51. Zhu Y, Knolhoff BL, Meyer MA, Nywening TM, West BL, Luo $\mathrm{J}$, et al. CSF1/CSF1R blockade reprograms tumor-infiltrating macrophages and improves response to T-cell checkpoint immunotherapy in pancreatic cancer models. Cancer Res 2014;74: 5057-5069.

52. Vicente E, Quijano Y, Ielpo B, Duran H. Total pancreatoduodenectomy en bloc with superior mesenteric artery and vein resection after gemcitabine and nab-paclitaxel neoadjuvancy. Surg Oncol 2017;26:276-277.

53. Takahashi S, Ohno I, Ikeda M, Kobayashi T, Akimoto T, Kojima $\mathrm{M}$, et al. Neoadjuvant S-1 with concurrent radiotherapy followed by surgery for borderline resectable pancreatic cancer: study protocol for an open-label, multicentre, prospective phase II trial (JASPAC05). BMJ Open 2017;7:e018445.

54. Fujii T, Yamada S, Murotani K, Kanda M, Sugimoto H, Nakao $\mathrm{A}$, et al. Inverse probability of treatment weighting analysis of upfront surgery versus neoadjuvant chemoradiotherapy followed by surgery for pancreatic adenocarcinoma with arterial abutment. Medicine (Baltimore) 2015;94:e1647.

55. Ielpo B, Caruso R, Duran H, Diaz E, Fabra I, Malavé L, et al. A comparative study of neoadjuvant treatment with gemcitabine plus nab-paclitaxel versus surgery first for pancreatic adenocarcinoma. Surg Oncol 2017;26:402-410.

56. Dholakia AS, Hacker-Prietz A, Wild AT, Raman SP, Wood LD, Huang P, et al. Resection of borderline resectable pancreatic cancer after neoadjuvant chemoradiation does not depend on improved radiographic appearance of tumor-vessel relationships. J Radiat Oncol 2013;2:413-425.

57. Rahman SH, Urquhart R, Molinari M. Neoadjuvant therapy for resectable pancreatic cancer. World J Gastrointest Oncol 2017;9: 457-465.

58. Assifi MM, Lu X, Eibl G, Reber HA, Li G, Hines OJ. Neoadjuvant therapy in pancreatic adenocarcinoma: a meta-analysis of phase II trials. Surgery 2011;150:466-473.

59. Nitsche U, Wenzel P, Siveke JT, Braren R, Holzapfel K, Schlitter AM, et al. Resectability after first-line FOLFIRINOX in initially unresectable locally advanced pancreatic cancer: a single-center experience. Ann Surg Oncol 2015;22 Suppl 3: S1212-S1220

60. Paniccia A, Edil BH, Schulick RD, Byers JT, Meguid C, Gajdos $\mathrm{C}$, et al. Neoadjuvant FOLFIRINOX application in borderline resectable pancreatic adenocarcinoma: a retrospective cohort study. Medicine (Baltimore) 2014;93:e198. 
61. Christians KK, Tsai S, Mahmoud A, Ritch P, Thomas JP, Wiebe L, et al. Neoadjuvant FOLFIRINOX for borderline resectable pancreas cancer: a new treatment paradigm? Oncologist 2014;19: 266-274.

62. Tinchon C, Hubmann E, Pichler A, Keil F, Pichler M, Rabl H, et al. Safety and efficacy of neoadjuvant FOLFIRINOX treat- ment in a series of patients with borderline resectable pancreatic ductal adenocarcinoma. Acta Oncol 2013;52:1231-1233.

63. Hosein PJ, Macintyre J, Kawamura C, Maldonado JC, Ernani V, Loaiza-Bonilla A, et al. A retrospective study of neoadjuvant FOLFIRINOX in unresectable or borderline-resectable locally advanced pancreatic adenocarcinoma. BMC Cancer 2012;12:199. 